\title{
Divulgação científica: uso social do produto dos estudos científicos na Fundação Joaquim Nabuco
}

\author{
Suiany Carvalho Padilha \\ Mestre em Gestão Pública; Fundação Joaquim Nabuco; \\ suiany.padilha@fundaj.gov.br \\ Nadi Helena Presser \\ Doutora em Engenharia de Produção; Universidade Federal de Pernambuco \\ nadihelena@uol.com.br \\ Alexandre Zarias \\ Doutor em Sociologia; Fundação Joaquim Nabuco \\ alexandre.zarias@fundaj.gov.br
}

Resumo: Este estudo identificou a trajetória percorrida pela informação científica produzida na Diretoria de Pesquisas Sociais (Dipes) da Fundação Joaquim Nabuco (Fundaj), no período de 2004 a 2013, observando os meios utilizados, as formas de apresentação e os atores envolvidos. O diagnóstico utilizou como parâmetro o ambiente de cultura científica apresentado pela espiral criada por Vogt (2003), configurando o cenário da Fundaj a partir da análise de documentos institucionais e de informações obtidas por meio de entrevistas semiestruturadas, realizadas com pesquisadores e gestores da Instituição. Embora a Fundaj apresente potencial institucional de cultura científica, este estudo apontou que parte das atividades é realizada por iniciativas individuais. Essa constatação é um aspecto importante deste estudo, pois a expressão "cultura científica", adotada por Vogt (2003), pressupõe uma concepção de construção de conhecimento científico, desde seu nascimento até sua ampla divulgação, visando ao seu uso social.

Palavras-chave: Divulgação científica. Cultura científica. Fundação Joaquim Nabuco.

\section{Introdução}

O conhecimento científico é um produto social, cultural e político e, por isso, deve ser democratizado e compartilhado, em favor do desenvolvimento humano. A sua disseminação contribui na promoção da qualidade de vida e para a inclusão social, uma vez que fornece as bases para a formação do pensamento crítico que, consequentemente, influencia na tomada de decisão e na construção do saber. O compartilhamento de informações ocorre através dos diversos meios de comunicação, com a 
participação de profissionais de múltiplas áreas do conhecimento, visando a uma ampla abrangência.

A partir do século XX o conceito de divulgação científica (DC) vem ganhando amplitude no que diz respeito à sua abrangência, porém requer veiculação diferenciada para alcançar os leitores que não integram a comunidade científica. Vogt (2006, p. 24) define a DC como um processo dinâmico e afirma que

[...] a expressão cultura científica nos soa mais adequada do que as várias outras tentativas de designação do amplo e cada vez mais difundido fenômeno da divulgação científica e da inserção no dia-a-dia de nossa sociedade dos temas da ciência e da tecnologia.

Este artigo reflete e discute a atual situação da Fundação Joaquim Nabuco (Fundaj) como promotora da cultura científica e apresenta um diagnóstico sobre a situação da DC na esfera de atuação da Diretoria de Pesquisas Sociais (Dipes), verificando a trajetória percorrida pela informação científica produzida, na perspectiva do modelo de cultura científica criado por Vogt (2003), o qual amplia a noção do significado de DC.

Por tudo isso, uma das contribuições mais significativas deste estudo está na sua dimensão social, pois a natureza de instituição pública da Fundaj pressupõe ações administrativas sob a égide da ética e da razão, objetivando sempre retornar à sociedade o produto do investimento público, para que, assim, a instituição fomente o uso social da informação científica como uma forma de contribuir para o desenvolvimento social.

O direito à informação, resguardado pela Constituição Federal, torna obrigatório aos órgãos públicos incluir na sua rotina a publicização das informações produzidas no âmbito institucional. Considerando a importância da contribuição com o desenvolvimento social, a Fundaj, instituição pública de pesquisas sociais criada no ano de 1949, pela Lei $\mathrm{n}^{\circ} 770$, de 21 de julho, atualmente vinculada ao Ministério da Educação (MEC), tem a finalidade de promover estudos e pesquisas no campo das Ciências Sociais e, também, o dever de disponibilizar o acervo de sua produção científica, favorecendo a democratização do conhecimento e estabelecendo elos entre a produção do conhecimento científico e a sociedade, numa contribuição efetiva com o desenvolvimento regional. 


\section{Procedimentos metodológicos}

A pesquisa de natureza aplicada se constituiu em um estudo de caso realizado na Fundaj, e suas análises tomaram como parâmetro a espiral da cultura científica de Vogt (2003). Designaram-se análises qualitativas, as quais, suportadas por dados quantitativos, permitiram traçar um diagnóstico do horizonte da cultura científica da instituição.

Documentos institucionais arquivados nas bases locais da Fundaj se constituíram como a base empírica da pesquisa, entre os mais expressivos estão os relatórios de gestão, memórias editoriais, clipagens, currículos Lattes dos pesquisadores, boletins e planos anuais de trabalho.

Entrevistas semiestruturadas auxiliaram na coleta e interpretação de dados e esclareceram informações não encontradas em registros documentais. Foram entrevistados dez pesquisadores, um deles em entrevista concedida oralmente e nove por meio de e-mail. Todos os respondentes estavam envolvidos com a produção e divulgação do conhecimento científico gerado na instituição. Vale salientar que no rol dos entrevistados havia alguns gestores que participaram ativamente no processo de gestão da Dipes/Fundaj no período estudado, 2004 a 2013. O conteúdo das entrevistas contemplou a opinião dos pesquisadores sobre o significado da DC e, ainda, a percepção dos mesmos sobre a política de DC da Fundaj desenvolvida no período de 2004 a 2013.

Apesar de o estudo ser delimitado por um recorte temporal de dez anos, ações realizadas em outras épocas são relevantes na construção de uma cultura científica. Sendo assim, foram consideradas algumas ações implementadas em períodos anteriores.

Este estudo, sem pretender ser exaustivo, encontrou algumas dificuldades que representaram limitações. Os documentos estudados, por não guardarem as mesmas características de conteúdo e forma, dificultaram ou impediram, muitas vezes, a análise minuciosa e uniforme do período estudado. Apresenta-se, ainda, nesse rol, o fato de que a Fundaj abriga um imenso potencial de memória institucional viva sem registro documental ou registrada desordenadamente, quando não mantida em arquivos "individuais", que, para serem acessados, dependem da disponibilidade 
dos seus detentores. O período de dez anos delimitado para a verificação foi definido, também, em consequência dessa dificuldade e falta de informações disponíveis.

\section{A divulgação científica}

O conhecimento tornou-se o eixo principal em torno do qual vem se modelando a sociedade pós-moderna. $\mathrm{O}$ avanço tecnológico permite que a informação chegue à população instantaneamente, mantendo-a em constante atualização e crescente nível de exigência, repercutindo na demanda de ações mais eficientes dos serviços públicos, sob os critérios de ética e sustentabilidade, os quais, atualmente, são disseminados e discutidos em âmbito mundial.

Nesse contexto, percebe-se a necessidade de gerir o conhecimento buscando novos paradigmas a serem enfrentados pela administração pública. A preocupação deve estar direcionada para a utilização dos recursos e disseminação das informações, “[...] com o desenvolvimento sustentável e com a busca de uma inteligência coletiva capaz de assegurar a melhoria das condições humanas, ambientais e sociais, no bojo da implantação da Gestão do Conhecimento em organizações públicas." (BENZ; SICSÚ, 2009, p. 220).

Atualmente, a literatura apresenta muitos conceitos e interpretações sobre a DC. O tema, por ultrapassar diferentes fronteiras da comunicação, adquire interpretações e olhares sob o foco das múltiplas áreas que abraça. Segundo Massarani (1998, p. 19), no Brasil, "[...] a designação 'divulgação científica', que já surgira no século passado, é hegemônica [...]", pois é frequentemente usada por revistas de divulgação da ciência, aparecendo em teses e dissertações que tratam do assunto. Sabe-se que a DC abrange todo e qualquer processo ou recurso utilizado para veiculação de informações científicas e tecnológicas (BUENO, $1985^{1}$ apud NASCIMENTO, 2008), direcionado para especialistas ou para o público em geral. Usando a terminologia "popularização da ciência", Mueller (2002, p. 1) defende a importância da divulgação do conhecimento científico para o público leigo e enfatiza a necessidade desse conhecimento ser amplamente disponibilizado para acesso do cidadão, adequando-se a linguagem ao público receptor. 
Comunicar é papel fundamental no processo da DC e, por isso, o pesquisador não pode ignorar a necessidade de uma boa comunicação para que o conhecimento científico atinja o seu uso social. Assim, há de se considerar a importância de parcerias com o jornalismo científico nesse processo de popularização da ciência.

\begin{abstract}
A saída é buscar uma aproximação entre todos aqueles que estão (ou deveriam estar) comprometidos com a democratização do conhecimento, objetivando o estabelecimento de parcerias, a definição de estratégias de atuação, a capacitação de fontes, o fortalecimento da educação fundamental e o debate amplo sobre o papel da ciência e da tecnologia numa sociedade em desenvolvimento. (BUENO, 2002, p. 230).
\end{abstract}

Reis (1968² apud KREINZ; PAVAN, 1998, p. 113) define a DC como "[...] a veiculação em termos simples da ciência como processo, dos princípios nela estabelecidos, das metodologias que emprega.". Reis (1968) busca traduzir a linguagem científica de forma que possa ser compreendida pelo público em geral, construindo uma relação de familiaridade entre a sociedade e a ciência, para que o cidadão tenha participação ativa e subsídios para analisar as situações criticamente e traçar seus próprios caminhos. Seguindo nessa linha, Calsamiglia (1997) concebe o sentido da ciência somente se for levada ao conhecimento dos cidadãos, e conceitua a DC, de forma geral, como "[...] o processo pelo qual se faz chegar a um grande público leigo o saber produzido por especialistas em uma disciplina científica." (CALSAMIGLIA, 1997, p. 1, tradução nossa) ${ }^{3}$.

Novos meios de DC surgiram na década de 80, época na qual foram criadas seções nos jornais diários, programas de TV e revistas para divulgação da ciência. Com a inovação tecnológica, os periódicos ganharam, também, versões eletrônicas na Internet, aumentando, assim, as possibilidades de atingir um público mais amplo. Ainda nessa década e na seguinte, começaram a surgir os centros, museus de ciência e instituições com a finalidade de popularizar a ciência, a exemplo do Centro de Divulgação Científica e Cultural, em 1980, em São Carlos; do Espaço Ciência Viva, em 1982, no Rio de Janeiro; e do Espaço Ciência, em Olinda, em 1994.

Acompanhando a tendência de investimento na popularização da ciência, o Laboratório de Estudos Avançados em Jornalismo (Labjor), criado pela Universidade de Campinas (Unicamp) em 1994, instituiu o curso em Jornalismo Científico em 1999, destinado à "[...] formação de divulgadores de ciência e assessores de comuni- 
cação de universidades e centros de pesquisa.". O curso objetiva "[...] capacitar jornalistas profissionais e cientistas para a divulgação científica, com a intenção de tornar público o debate sobre ciência e tecnologia $(\mathrm{C} \& \mathrm{~T})$ e reduzir a distância entre o conhecimento científico e o cotidiano das pessoas." (UNIVERSIDADE..., 2014). Considerado centro de referência na formação e nos estudos em divulgação científica no Brasil e na América Latina, o Labjor, em plena atividade, possui revistas impressas e eletrônicas que contribuem efetivamente com a divulgação da ciência no país, como as revistas ComCiência, em parceria com a Sociedade Brasileira para o Progresso da Ciência (SBPC), e Ciência e Cultura, da SBPC.

A atividade de pesquisa científica em favor do desenvolvimento científico e tecnológico no Brasil ganhou importância significativa na agenda do governo na década de 50, quando foram criadas as instituições de fomento Conselho Nacional de Desenvolvimento Científico e Tecnológico (CNPq) e Coordenação de Aperfeiçoamento de Pessoal e Nível Superior (CAPES). A busca por uma maior autonomia na área de C\&T, na década de 70, levou a agenda pública a pensar em ações para o desenvolvimento da ciência e tecnologia. Em 1975, durante o regime militar, foi instituído o Sistema Nacional de Desenvolvimento Científico e Tecnológico (SNDCT), dentro das ações do I Plano Nacional de Desenvolvimento (I PND). Era o início dos chamados Planos Básicos de Desenvolvimento Científico e Tecnológico, administrados pelo CNPq, que tinham como objetivo central a busca da autossuficiência científica e tecnológica nacional.

Após o período militar, a agenda do novo governo priorizou, dentre outras ações, o desenvolvimento científico e tecnológico, criando o Ministério da Ciência e Tecnologia (MCT) e estabelecendo ações para popularização da produção científica nacional, que tiveram como marco histórico a realização das Conferências Nacionais de Ciência e Tecnologia, em 1985, 2001, 2005 e 2010.

Dando continuidade às atividades de desenvolvimento e difusão científica, em 2012, o Ministério da Ciência, Tecnologia e Inovação (MCTI) elaborou um documento chamado "Estratégia Nacional para Ciência, Tecnologia e Inovação (ENCTI) 2012-2015", que iria nortear as ações governamentais, destacando a "importância da ciência, tecnologia e inovação (CT\&I)" como eixo estruturante do desenvol- 
vimento do país. Nesse documento foi criada a diretriz "CT\&I para o Desenvolvimento Social", enfatizando que:

Destaca-se entre as principais preocupações da ENCTI sua contribuição para o desenvolvimento social do país. A apropriação do conhecimento científico e tecnológico pela sociedade permite, entre outras coisas, a ampliação da cidadania com base em informações robustas, $o$ incremento na renda, por meio da aplicação e utilização de práticas comprovadas e a melhoria da qualidade de vida. No âmbito desse programa prioritário será dada ênfase a três vertentes principais: (i) popularização da C,T\&I e melhoria do ensino da ciências; (ii) inclusão produtiva e social; e (iii) tecnologias para cidades sustentáveis. (BRASIL, 2012, p. 82 , grifo nosso).

É importante mencionar, ainda, como instrumento regulador do tema, a recente Lei de Acesso à Informação no Brasil - Lei $n^{\circ}$ 12.527, de 18/11/2011, que assegura ao cidadão, a partir de uma gestão transparente nos órgãos públicos, o direito fundamental de acesso a toda e qualquer informação pública, através dos meios viabilizados pela tecnologia da informação, e que contribui para a formação de uma sociedade com opinião, capaz de buscar seus direitos e a informação que lhe seja útil para uma qualidade de vida melhor.

Percebe-se a permanência do tema ao longo de várias décadas, numa evolução contínua e evidente de se reconhecer a necessidade prioritária do investimento no ensinar e no fazer circular o conhecimento científico, buscando seu uso social, contribuindo, assim, efetivamente, para um salto qualitativo do desenvolvimento do país.

Hoje, a sociedade está cercada pela ciência e pela tecnologia, devendo, portanto, ir em busca da conquista da absorção natural dessas áreas no cotidiano. Nesse momento, diante de uma sociedade conectada em rede, onde a informação circula amplamente através dos mais diversos espaços e meios de comunicação, a DC exerce indispensável papel, uma vez que se torna o elo entre o conhecimento e o cidadão, proporcionando sua participação ativa, ou seja, sua inserção no processo de produção, de difusão e de ensino e aprendizagem da ciência. 


\title{
4 Cultura científica
}

Vogt (2003) cunhou a expressão "cultura científica" como um termo mais amplo e adequado para denominar a divulgação científica e a inclusão da ciência e tecnologia no meio social. Para Vogt (2003), o termo "cultura" abrange o processo desde o nascimento até a disseminação e utilização do conhecimento produzido e busca envolver o cidadão com o mundo científico:

[...] a ideia de que o processo que envolve o desenvolvimento científico é um processo cultural, quer seja ele considerado do ponto de vista de sua produção, de sua difusão entre pares ou na dinâmica social do ensino e da educação, ou ainda do ponto de vista de sua divulgação na sociedade, como um todo, para o estabelecimento das relações críticas necessárias entre o cidadão e os valores culturais, de seu tempo e de sua história. (VOGT, 2003, p. 2).

O autor estudou a dinâmica da cultura científica e o ponto convergente entre arte e ciência, considerando ambas geradoras de conhecimento, sendo a primeira pela metáfora e sensibilização do conceito e a segunda pela demonstração lógica e experiência.

No prefácio do livro When science becomes culture, que agrega trabalhos apresentados no simpósio internacional sobre cultura científica, em abril de 1994, em Montreal, Canadá, Berlinguet (1994) escreve:

\begin{abstract}
No passado, o pequeno grupo de cientistas que, com grande dificuldade, examinou as primeiras leis do nosso universo estava cercado pela sociedade. Com a expansão do conhecimento, nas palavras de Pierre Fayard, houve "uma revolução copernicana que tende a fazer a ciência circular em torno do público, e não o contrário". Hoje, quer queiramos ou não, estamos cercados em nosso dia a dia pela ciência e pela tecnologia. Portanto, é melhor tentar conquistá-las do que permanecer passivo diante de seus desenvolvimentos. (BERLINGUET, 1994, doc. não paginado, tradução nossa).
\end{abstract}

Vogt (2006, p. 25), ao citá-lo em seus escritos, questiona: "como é possível realizar essa conquista sem estar envolvido diretamente no processo de produção, de difusão e de ensino e aprendizagem da ciência?". E conclui:

A resposta é "pela divulgação científica", isto é, pela participação ativa do cidadão nesse amplo e dinâmico processo cultural em que a ciência e 


\begin{abstract}
a tecnologia entram cada vez mais em nosso cotidiano, da mesma forma que a ficção, a poesia e a arte fazem parte do imaginário social e simbólico de nossa realidade e de nossos sonhos, multiplicando em nossa existência única, e provisória, a infinitude de vidas e vivências que vivemos sem jamais tê-las vivido.
\end{abstract}

Essa dinâmica pode ser visualizada através da Espiral da Cultura Científica (Figura 1) criada por Vogt (2003). A curva do conhecimento, em movimento espiral, ganha amplitude na relação tempo e espaço (eixo vertical) e estabelece elos na troca do saber entre atores e meios diferentes. Observa-se que cada quadrante busca atingir um público específico, com diferentes interesses e necessidades no consumo do conhecimento. A curva se desenvolve em sentido horário quando a descoberta nasce e é discutida entre pares, ganha amplitude quando o conhecimento estabelecido é repassado através do ensino da ciência, é apresentada do ensino para ciência e finaliza o ciclo com a ampla divulgação para a sociedade em geral. A curva retorna sempre a um ponto mais "[...] alargado de conhecimento e de participação da cidadania no processo dinâmico da ciência e de suas relações com a sociedade [...]" (VOGT, 2003, p. 6). 
Figura 1 - Espiral da Cultura Científica

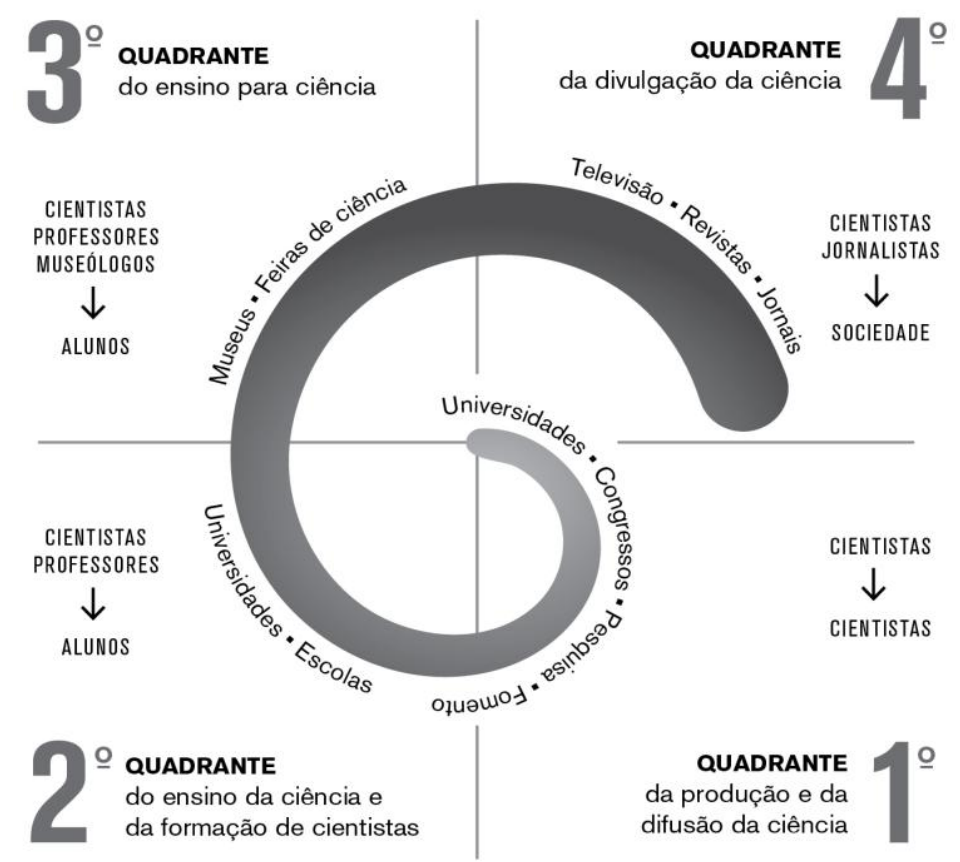

Fonte: Adaptado de Vogt (2003).

Para cada quadrante existem diversos grupos de destinadores e destinatários. Faz-se necessário observar as variáveis que exercem influência na disseminação da informação (linguagem, instrumento, meios, conteúdo, abordagem). De um quadrante para o outro deverá haver uma perda gerenciada de conteúdo para que a informação chegue adequadamente a certo público, servindo aos interesses da comunidade. Vogt (2003, p. 6) conclui que "[...] a espiral da cultura científica pretende representar, na forma que lhe é própria, e, em termos gerais, a dinâmica constitutiva das relações inerentes e necessárias entre ciência e cultura.".

A pesquisa se concretiza através da informação científica, que perde o sentido de ser quando não é utilizada e aplicada. "Fluido precioso, continuamente produzido e renovado, a informação só interessa se circula, e, sobretudo, se circula livremente." (LE COADIC, 2004, p. 26). É condição de existência da informação científica que sejam adotadas ações para que esta circule e seja amplamente divulgada, disseminada entre e para as pessoas. Essa circulação deve se dar desde os primeiros ensaios de inserção ao mundo do conhecimento, na escola, para que a vontade do saber, do conhecer, se torne um hábito para o cidadão.

A Fundaj, na perspectiva de uma instituição voltada para a produção e difusão do conhecimento científico, tem por missão: 
[...] gerar conhecimento no campo das humanidades com a finalidade de atender a demandas e necessidades relacionadas a educação e cultura, compreendidas de forma interdependente, com vistas ao desenvolvimento justo e sustentável da sociedade brasileira. (BRASIL, doc. eletrônico, 2014).

Por conseguinte, busca colaborar com a preservação e a difusão de valores científicos e culturais, com a formação e qualificação profissional em diferentes áreas, inserindo-se, estrategicamente, nos quatro quadrantes da espiral proposta por Vogt. A partir de suas ações, sedimentadas pelos mais de 60 anos de tradição em pesquisa, cultura e educação, que contribuem efetivamente para a formação de uma cultura científica e promoção da ciência, tecnologia e inovação, disponibilizando-as para o grande público, a Fundaj foi vencedora da $32^{\text {a }}$ edição do Prêmio José Reis de Divulgação Científica e Tecnológica - 2012.

O Prêmio José Reis, promovido anualmente pelo Conselho Nacional de Desenvolvimento Científico e Tecnológico (CNPq) e pelo Ministério da Ciência, Tecnologia e Inovação (MCTI), é atribuído a personalidades e instituições que contribuem para a formação de uma cultura científica e pela difusão da Ciência, Tecnologia e Inovação. Inserida nesse contexto de novas ações para o desenvolvimento do Nordeste, a Instituição utiliza-se dos diversos meios tecnológicos e recursos estruturais disponíveis para o amplo e fácil acesso da população ao conhecimento que produz e armazena, por meio de uma gestão pública inovadora focada no desenvolvimento da sociedade. Em 2012, a Fundaj participou do Prêmio José Reis apresentando os seguintes produtos, atividades e espaços que guardam relação com a concepção de Cultura Científica proposta por Vogt: a revista eletrônica Coletiva, o portal Pesquisa Escolar Online, a Editora Massangana, a Massangana Multimídia Produções, o espaço cultural Engenho Massangana, o Museu do Homem do Nordeste e a participação anual na Semana Nacional de Ciência e Tecnologia.

\section{Análise do cenário sob a perspectiva da espiral da cultura científica}

A Fundaj nasceu em 1949, a partir da criação do Instituto Joaquim Nabuco, pelo sociólogo Gilberto Freyre, tendo como objetivo central a pesquisa social. No decorrer do tempo, sua estrutura passou por reconfigurações e, atualmente, conta com três diretorias: Diretoria de Formação e Desenvolvimento Profissional (DIFOR), Direto- 
ria de Memória, Educação, Cultura e Arte (MECA) e Diretoria de Pesquisas Sociais (DIPES), esta última permanecendo em preservação da área finalística de pesquisas social e das origens da Instituição, cenário do estudo.

A Dipes/Fundaj, atualmente área de maior concentração de pesquisas na Instituição, contempla, em seus objetivos, a divulgação dos resultados das suas pesquisas à sociedade brasileira e possui a estrutura representada na Figura 2.

Figura 2 - Estrutura organizacional da Dipes/Fundaj

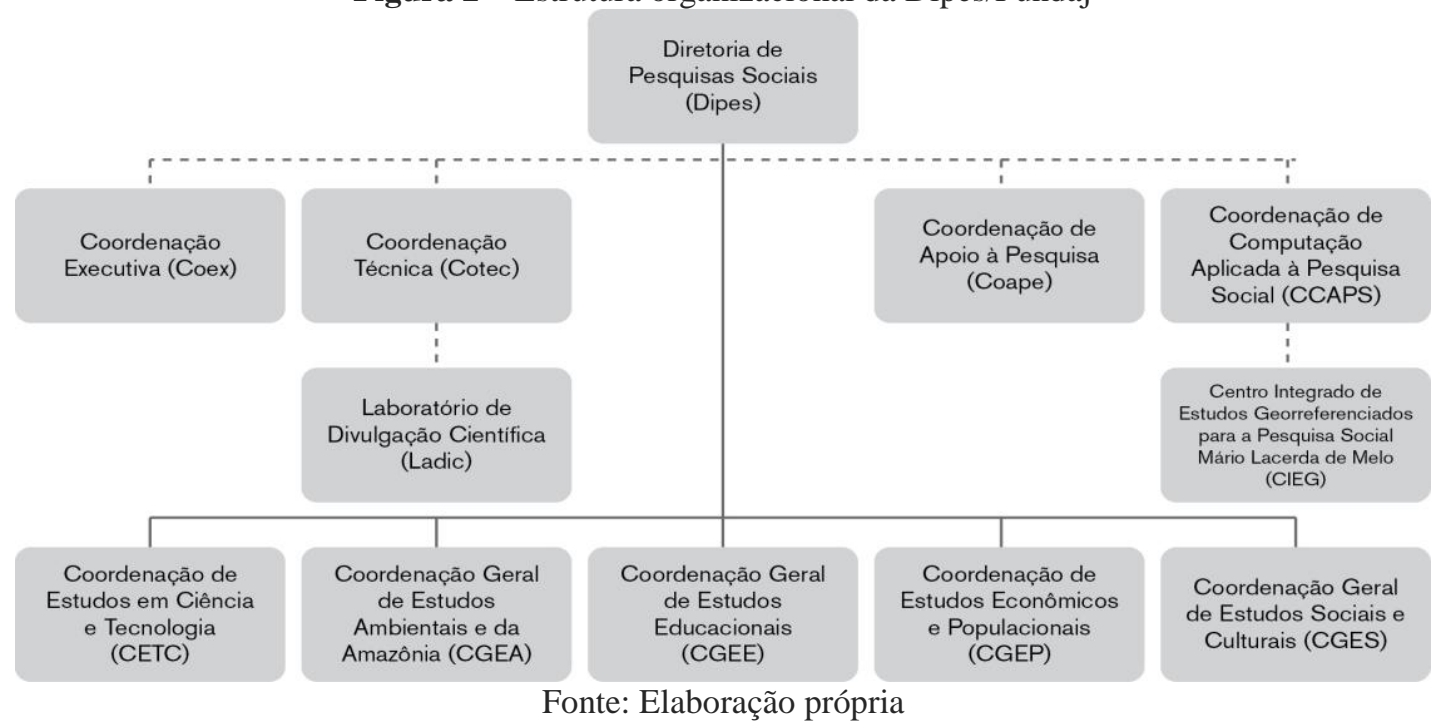

Os registros documentais totalizam a conclusão de 114 pesquisas realizadas pela Dipes/Fundaj no período estudado (2004-2013) e um corpo composto por 26 doutores, 27 mestres e quatro especialistas em 2013, entre as cinco coordenações de pesquisa e a unidade central. O levantamento de dados realizado na Dipes/Fundaj, somado às propostas já elaboradas para criação de uma política de DC, permitiu a construção de um cenário inserido na Espiral da Cultura Científica (VOGT, 2003). As ações ou propostas da Diretoria de Pesquisas Sociais foram identificadas e apontadas em cada quadrante da espiral de acordo com os meios utilizados e públicos destinadores e destinatários.

Como pode ser verificado na Figura 3, a criação de uma cultura científica na Dipes/Fundaj é diagnosticada pela sua capacidade de empreender ações para públicos específicos, com diferentes interesses e necessidades no consumo do conhecimento. 
Figura 3 - Espiral da Cultura Científica na Dipes/Fundaj

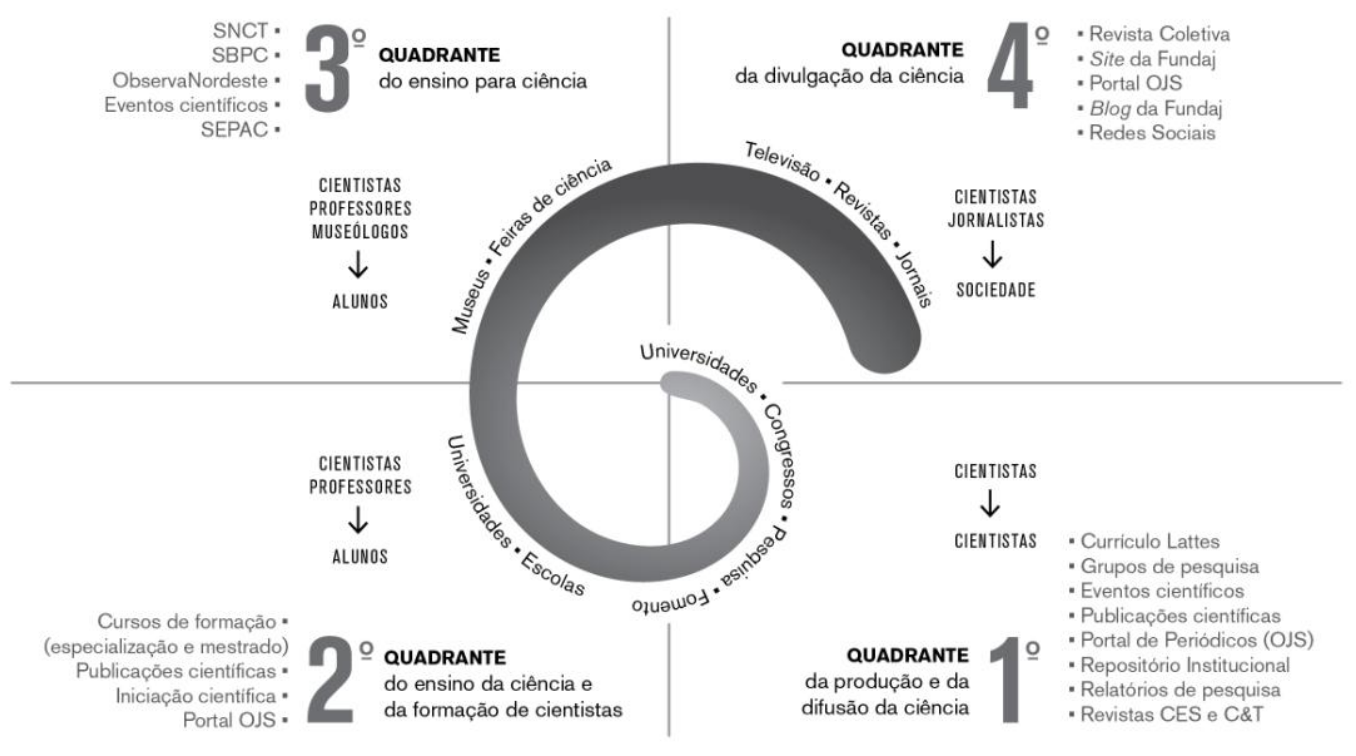

Fonte: Adaptado de Vogt (2003), elaboração própria com base na análise dos dados da Dipes/Fundaj.

A seguir, cada quadrante é apresentado nas suas constituições e especificidades, de modo a retratar com mais detalhes a situação atual da cultura científica na Dipes/Fundaj.

\subsection{Primeiro Quadrante}

É composto pelas ações que estão relacionadas à produção científica, onde os destinadores e destinatários são os especialistas (cientistas, pesquisadores, centros, laboratórios, universidades, instituições privadas ou governamentais). $\mathrm{Na}$ Dipes/Fundaj o cenário identificado é o que se segue.

\subsubsection{Currículo Lattes}

É utilizado pela maioria das instituições de pesquisa no país, se firmando como uma importante base de dados sobre a atividade científica no Brasil. É uma ferramenta que pode ser usada para dar visibilidade institucional a partir do potencial de profissionais da ciência que a instituição apresenta, como pode ser observado no Quadro 1, em relação à Dipes/Fundaj. 
Quadro 1- Situação dos pesquisadores da Dipes/Fundaj em relação à atualização do Currículo Lattes.

\begin{tabular}{|l|l|}
\hline $\begin{array}{l}\text { Situação verificada em } 25 \text { de novembro de } \\
\mathbf{2 0 1 3}\end{array}$ & $\begin{array}{l}\text { Situação verificada em 6 de junho de 2014 } \\
\text { (após solicitação de atualização aos pesqui- } \\
\text { sadores por parte da Fundaj) }\end{array}$ \\
\hline $\begin{array}{l}\text { 59\% do total de pesquisadores sem atualização } \\
\text { dos dados nos últimos seis meses. }\end{array}$ & $\begin{array}{l}38 \% \text { do total de pesquisadores continuaram } \\
\text { sem atualização dos dados. }\end{array}$ \\
\hline $\begin{array}{l}38 \% \text { do total de pesquisadores com os dados } \\
\text { atualizados até a consulta. }\end{array}$ & $\begin{array}{l}59 \% \text { do total de pesquisadores com os dados } \\
\text { atualizados até a consulta. }\end{array}$ \\
\hline $\begin{array}{l}4 \% \text { do total de pesquisadores não possuíam regis- } \\
\text { tro na Plataforma Lattes }\end{array}$ & $\begin{array}{l}4 \% \text { do total de pesquisadores continuaram } \\
\text { sem registro na Plataforma Lattes. }\end{array}$ \\
\hline
\end{tabular}

Fonte: Elaboração própria com base na análise da Plataforma Lattes (CNPq).

\subsubsection{Diretório dos Grupos de Pesquisa (CNPq)}

Ambiente virtual que abriga os grupos de pesquisa científica e tecnológica em atividade no país. É um banco de informações sobre o perfil da atividade científicotecnológica, tornando-se importante ferramenta para o planejamento, gestão e memória dessa atividade no Brasil. Foram identificados apenas cinco grupos de pesquisa da Dipes no diretório do CNPq, dos quais três estão em inatividade, retratando o baixo envolvimento acadêmico da pesquisa na Fundaj, conforme discriminado no Quadro 2.

Quadro 2 - Grupos de pesquisa da Fundaj no Diretório dos Grupos de Pesquisa no Brasil - CNPq (maio/2014)

\begin{tabular}{|l|l|l|l|}
\hline Grupo de pesquisa & Área & Início & Situação em maio/2014 \\
\hline Modernização tecnológica periférica & Economia & 1980 & $\begin{array}{l}\text { Não atualizado há mais } \\
\text { de doze meses }\end{array}$ \\
\hline Águas do Semiárido Nordestino & Agronomia & 1990 & $\begin{array}{l}\text { Não atualizado há mais } \\
\text { de doze meses }\end{array}$ \\
\hline Culturas populares: novos desafios & História & 1997 & $\begin{array}{l}\text { Não atualizado há mais } \\
\text { de doze meses }\end{array}$ \\
\hline Estado, cidadania e atores sociais & Ciência política & 1998 & Certificado \\
\hline $\begin{array}{l}\text { Políticas, programas e práticas na educa- } \\
\text { ção infantil }\end{array}$ & Educação & 2006 & Certificado \\
\hline
\end{tabular}

Fonte: Elaboração própria com base na análise do Diretório dos Grupos de Pesquisa no Brasil do CNPq. 


\subsubsection{Realização de eventos científicos}

No período estudado, os relatórios de gestão consultados (FUNDAÇÃO JOAQUIM..., 2007a; 2007b; 2012; 2013) apresentaram 294 realizações de eventos para difusão dos estudos científicos (seminários, workshops, palestras, festivais, cursos, encontros, conferências, simpósios e debates) produzidos pela Dipes/Fundaj, quase sempre voltados a um público específico. O Quadro 3 mostra a relação entre a realização de eventos científicos e o percentual dos recursos do Tesouro Nacional utilizados em cada ano.

Quadro 3 - Recursos do Tesouro Nacional destinados à Dipes/Fundaj, utilizados em eventos científicos realizados pela Dipes (2004-2013)

\begin{tabular}{|c|c|c|c|c|c|c|c|c|c|c|}
\hline \multicolumn{1}{|c|}{ Dados } & $\mathbf{2 0 0 4}$ & $\mathbf{2 0 0 5}$ & $\mathbf{2 0 0 6}$ & $\mathbf{2 0 0 7}$ & $\mathbf{2 0 0 8}$ & $\mathbf{2 0 0 9}$ & $\mathbf{2 0 1 0}$ & $\mathbf{2 0 1 1}$ & $\mathbf{2 0 1 2}$ & $\mathbf{2 0 1 3}$ \\
\hline $\begin{array}{l}\text { Número de } \\
\text { eventos } \\
\text { realizados }\end{array}$ & 16 & 22 & 13 & 64 & 53 & 36 & 23 & 29 & 21 & 17 \\
\hline $\begin{array}{l}\% \text { utilizada } \\
\text { dos recursos } \\
\text { do Tesouro } \\
\text { destinados à } \\
\begin{array}{l}\text { Dipes/ Fun- } \\
\text { daj }\end{array}\end{array}$ & $100 \%$ & $93,36 \%$ & $12 \%$ & $86 \%$ & $91,10 \%$ & $81,08 \%$ & $72,32 \%$ & $51,94 \%$ & $79,44 \%$ & $22,53 \%$ \\
\hline
\end{tabular}

Fonte: Elaboração própria com base na análise dos documentos da Dipes/Fundaj.

Os dados indicam que, apesar do aporte de recursos disponíveis, houve um número pequeno de eventos realizados, sofrendo uma queda significativa entre 2007 e 2013. A partir de 2010, os números apontam que a utilização dos recursos disponibilizados pelo Tesouro Nacional se manteve abaixo dos oitenta por cento.

\subsubsection{Participação em eventos científicos}

A participação de pesquisadores nesses eventos tem grande importância na DC da Dipes/Fundaj, trazendo repercussões positivas na visibilidade institucional. Os documentos consultados registraram a participação dos pesquisadores em eventos científicos nacionais e internacionais, conforme os números apontados no Quadro 4. 
Quadro 4 - Participação dos pesquisadores em eventos científicos no período 20112013

\begin{tabular}{|l|c|c|c|}
\hline \multicolumn{1}{|c|}{ Dados } & $\mathbf{2 0 1 1}$ & $\mathbf{2 0 1 2}$ & $\mathbf{2 0 1 3}$ \\
\hline Participação em eventos & 67 & 142 & 218 \\
\hline Pesquisadores participantes & 10 & 39 & 36 \\
\hline Pesquisadores em atividade no ano & 71 & 59 & 57 \\
\hline Proporção de pesquisadores participantes (\%) & $14 \%$ & $66 \%$ & $63 \%$ \\
\hline
\end{tabular}

Fonte: Elaboração própria com base na análise dos documentos da Dipes/Fundaj.

Os documentos só possibilitaram o exame dos dados a partir de 2011 e, nesse período, as análises revelaram que 14\%, 66\% e 63\% dos pesquisadores da Dipes/Fundaj em cada ano, 2011, 2012 e 2013, respectivamente, participaram de eventos científicos. Foi observada, ainda, uma concentração da atividade em $66 \%$ do total de pesquisadores em efetivo exercício no período, entenda-se que esse percentual abriga a participação de um mesmo grupo de pesquisadores efetivos no período. Isso significa dizer que $44 \%$ dos pesquisadores efetivos, nesses anos, não participaram dessa ação.

\subsubsection{Publicações científica}

A disponibilização dos resultados das pesquisas pode atender a dois tipos de público: especialista e não especialista. Para os especialistas do meio científico, essa disponibilização pode ser realizada através das publicações científicas: ações do primeiro quadrante; para o público leigo, a informação deve ser levada por uma linguagem adaptada: quarto quadrante da Espiral de Vogt (2003). O Quadro 5 apresenta o panorama anual da produção por pesquisador. Ressalta-se que o quantitativo em relação ao período 2004 a 2006 e de 2008 não constam nos relatórios e demais documentos analisados, impossibilitando a verificação do período completo que compreende 2004-2013. 
Quadro 5 - Publicações científicas dos pesquisadores do quadro efetivo da Fundaj por ano

\begin{tabular}{|l|c|c|c|c|c|c|}
\hline Dados & $\mathbf{2 0 0 7}$ & $\mathbf{2 0 0 9}$ & $\mathbf{2 0 1 0}$ & $\mathbf{2 0 1 1}$ & $\mathbf{2 0 1 2}$ & $\mathbf{2 0 1 3}$ \\
\hline Total de publicações & 150 & 103 & 132 & 93 & 138 & 37 \\
\hline Total de pesquisadores & 70 & 76 & 70 & 71 & 59 & 57 \\
\hline \% de pesquisadores que publicaram & $57 \%$ & $41 \%$ & $63 \%$ & $56 \%$ & $75 \%$ & $30 \%$ \\
\hline Média de publicação por pesquisador & 2,14 & 1,36 & 1,89 & 1,31 & 2,34 & 0,65 \\
\hline
\end{tabular}

Fonte: Elaboração própria com base na análise dos documentos da Dipes/Fundaj.

Os dados dos quadros apresentados e as entrevistas com os pesquisadores sinalizam que a realização dessa atividade é desvinculada de política científica institucional. Se existem metas a serem cumpridas por plano de carreira (Lei 8.691/93), estas carecem de devido monitoramento e gestão. Em uma análise mais detalhada (ver Quadro 6), fica visível a baixa a produção dos pesquisadores que estiveram em atividade de pesquisa nos últimos 6 anos, sendo verificada uma média geral inferior a dois artigos por ano: pouco mais da metade $(56 \%)$ realizaram cinco ou mais publicações, 35\% realizaram de uma a quatro publicações e 9\% não publicaram no período.

Quadro 6 - Publicações científicas dos pesquisadores da Dipes/Fundaj (2008-2013)

\begin{tabular}{|l|c|}
\hline Dados & Percentual \\
\hline Publicaram (1 a 4 publicações) & $35 \%$ \\
\hline Publicaram (5 ou mais publicações) & $56 \%$ \\
\hline Não publicaram & $9 \%$ \\
\hline
\end{tabular}

Fonte: Elaboração própria com base na análise dos documentos da Dipes/Fundaj.

\subsubsection{Portal de Periódicos (OJS)}

Disponibiliza virtualmente, para livre acesso, o conteúdo das revistas Ciência \& Trópico, Cadernos de Estudos Sociais e Horizontes LatinoAmericanos, publicadas pela instituição. 


\subsubsection{Revista Ciência \& Trópico (C\&T)}

Revista impressa com publicação semestral, de caráter interdisciplinar, apresenta matérias a partir de trabalhos especializados em ciências sociais, ciência e tecnologia e humanidades, com distribuição nacional e internacional. A Ciência \& Trópico é o seguimento do Boletim do Instituto Joaquim Nabuco de Pesquisas Sociais, que, segundo Freston (2001), foi o principal foro de divulgação das pesquisas. O Boletim foi criado em 1952, "[...] podendo reivindicar, então, o título de revista de maior duração na história das ciências sociais no Brasil." (FRESTON, 2001, p. 394). A Resolução $\mathrm{n}^{\circ}$ 267, de outubro de 1972, alterou seu título pelo sociólogo Gilberto Freyre, passando a ser denominado Ciência \& Trópico, como permanece até hoje. A revista está disponível no Portal de Periódicos Fundaj e possui, também, um perfil na rede social Facebook. A última publicação virtual foi o volume 37, número 2 (2013). Não foi encontrada classificação da referida revista nos anos e extratos de avaliação no Portal CAPES.

\subsubsection{Revista Cadernos de Estudos Sociais (CES)}

Revista impressa com publicação semestral que divulga trabalhos realizados por pesquisadores de universidades e instituições de pesquisa do Brasil e do exterior. Faz também publicação virtual através do Portal de Periódicos da Fundaj e utiliza um perfil criado na rede social Facebook para sua divulgação. A última publicação virtual foi o volume 29, número 2 (2014). A CES tem classificação Qualis B2 na área interdisciplinar e Qualis C na área de sociologia.

\subsubsection{Relatórios de pesquisa}

Apresentar os relatórios e resultados dos estudos científicos para o público especialista é necessário, mas disponibilizar para o público não especialista não é menos importante. Os relatórios finais constituem importante fonte para extração de informações a serem levadas à sociedade através de uma linguagem plenamente adaptada ao público leigo, que se concretiza no quarto quadrante da Espiral (VOGT, 2003). É 
quando a informação irá atingir o seu uso social. A disponibilização é necessária, ainda, para atender ao princípio da transparência pública, é uma prestação de contas à sociedade. A publicização dos produtos da atividade de pesquisa na Dipes/Fundaj se dá, efetivamente, no âmbito do primeiro quadrante, através da participação dos pesquisadores em congressos, seminários, publicação de artigos e livros etc.

\subsection{Segundo Quadrante}

O segundo quadrante contempla as ações de ensino e aprendizagem da ciência para estudantes e formação de cientistas, através dos profissionais de ensino. As atividades identificadas, conforme destacadas na Figura 3, são as seguintes:

\subsubsection{Programa Institucional de Bolsa de Iniciação Científica (Pibic)}

Iniciou em 2004 e contou com 26 estudantes em 2013. O Pibic, dentro das suas atividades, realiza anualmente a Jornada de Iniciação Científica, que tem por objetivo a apresentação dos estudos desenvolvidos pelos estudantes.

\subsubsection{Cursos de formação}

A Dipes/Fundaj trabalhou em parceria com as demais diretorias finalísticas da Instituição na implantação de cursos de curta duração, especialização e mestrado.

\subsection{Terceiro Quadrante}

Ultrapassando a fronteira do universo delimitado por ensino e aprendizagem, o terceiro quadrante leva a informação científica para um público mais abrangente. O conhecimento parte de cientistas, pesquisadores e gestores ligados à área de produção científica e cultural, tanto para um público de estudantes e aprendizes como para um público leigo, num processo de busca da aceitação da ciência. Na Dipes/Fundaj identificaram-se as seguintes ações: 


\subsubsection{Semana Nacional de Ciência e Tecnologia (SNCT)}

Esse evento nacional acontece desde 2004, promovido pelo MCTI, com o objetivo de mobilizar a população, principalmente crianças e jovens, em torno de temas e atividades de C\&T. O evento é realizado em todo o país por diversas instituições. A SNCT conta com o envolvimento de pesquisadores das diversas coordenações da Dipes e outras áreas da Fundaj, contribuindo com palestras, exposições, minicursos e debates.

\subsubsection{Reunião Anual da Sociedade Brasileira para o Progresso da Ciência (SBPC)}

É um fórum de difusão dos avanços da ciência nas diversas áreas do conhecimento, tendo realizado em 2014 sua $66^{\text {a }}$ edição. A Fundaj participou do evento em 2012, a 64 $4^{\mathrm{a}}$ reunião, realizada na cidade de São Luís, com a conferência "A divulgação científica no Nordeste e o papel da Fundação Joaquim Nabuco", ministrada pelo então Presidente, Fernando José Freire. A Instituição marcou presença, também, na $65^{\mathrm{a}}$ reunião, em 2013, na cidade de Recife, por meio da exposição de suas revistas científicas ( $C \& T$, CES e Coletiva) e com a participação de pesquisadores como palestrantes, debatedores, instrutores em conferências, simpósios, mesas-redondas, minicursos e, ainda, com a participação de estagiários na exposição de pôsteres.

\subsubsection{Observatório Social do Nordeste (ObservaNordeste)}

Constitui um fórum permanente de acompanhamento, reflexão e debates qualificados sobre a realidade social da Região Nordeste, voltado a pesquisadores, intelectuais, estudantes universitários e militantes políticos e todo público interessado nos movimentos sociais brasileiros, operando tanto em ambiente virtual como físico, com a realização de simpósios. O conteúdo é disponibilizado através do site da Fundaj, utilizando, ainda, veículos de publicação impressos (boletins, revistas etc.). 


\subsubsection{Seminário Permanente de Pesquisa e Atualização (SEPAC)}

Programa de difusão, prospecção e atualização do conhecimento nas ciências sociais, vem sendo realizado desde 2006 e é aberto ao público em geral.

\subsection{Quarto Quadrante}

O quarto quadrante, onde se concretiza a DC em toda sua amplitude, se constitui das atividades próprias da divulgação científica e tem como destinadores a mais os profissionais de jornalismo. Esses, os divulgadores científicos, se encarregam da transmissão direcionada a um público amplo e abrangente, utilizando-se dos mais diversos meios de comunicação. Tem-se como destinatária toda a sociedade "[...] organizada em suas diferentes instituições, inclusive, e principalmente, as da sociedade civil [...]" (VOGT, 2003, p. 5), o que torna o cidadão o destinatário principal dessa interlocução da cultura científica. É o instante da popularização do conhecimento para o uso social. Este estudo identificou ações próprias desse quadrante e canais já existentes, conforme se segue.

\subsubsection{Homepage}

Atualmente está desatualizada, pois não há ações sistemáticas que estabeleçam elos entre as áreas de pesquisa e os responsáveis que alimentam esse site.

\subsubsection{Blog da Fundação}

Pode ser acessado pelo site oficial, entrementes se constate a publicação de um conteúdo referente apenas às atividades culturais da instituição.

\subsubsection{Redes sociais}

Utilizadas para a publicação virtual das revistas $C E S$ e $C \& T$ e divulgação de eventos, embora ainda persista seu uso individualizado pelos membros de setores da 
Fundaj, evidenciando a prática de ações fragmentadas, desvinculadas de um plano institucional.

\subsubsection{Laboratório de Divulgação Científica (Ladic)}

Seu objetivo é criar uma infraestrutura mínima de publicação e divulgação científica e, portanto, promover DC nos meios impresso e virtual. Assim, o Ladic é o espaço que deve estabelecer o elo entre a produção científica e sua veiculação nos diversos meios virtuais e impressos. Entretanto, além da carência de recursos humanos, este estudo apontou a inexistência de institucionalização para que as diferentes áreas (setor de comunicação e TI) atuem conjuntamente.

\subsubsection{Revista Coletiva}

A revista eletrônica de DC visa oferecer um enfoque crítico sobre as atividades científica e cultural, da produção e uso do conhecimento em diversas áreas do saber; teve uma média diária de 129, 301 e 935 acessos nos anos de 2011, 2012 e 2013, respectivamente, de origem nacional e internacional, sendo $93,08 \%$ das visitas ao seu site realizadas a partir de território brasileiro.

\section{Considerações finais}

Após percorrer a trajetória para construção da realidade na Dipes/Fundaj, chega-se a uma situação que permite um olhar direcionado a atingir o interesse da análise: a verificação do caminho percorrido pela informação científica da Dipes/Fundaj, no período de 2004 a 2013, observando ações realizadas, os meios utilizados, as formas de apresentação e os atores envolvidos.

A amplitude do conceito de cultura científica concebido por Vogt (2003) permite a análise de instituições de pesquisa na diversidade de suas ações. Sob a ótica da cultura científica, a produção do conhecimento considera os aspectos históricos, culturais e sociais, ultrapassando os limites do foco apenas no conteúdo, constituindo-se, assim, em um processo que abrange desde o momento da produção do 
conhecimento até a sua difusão entre os diversos atores envolvidos, através dos diversos e abrangentes canais de comunicação. A verificação desse processo nas instituições de pesquisa pode ser realizada através da produtividade dos pesquisadores e difusão da produção em eventos científicos, do envolvimento com atividades de formação profissional e, ainda, através do envolvimento dos pesquisadores em ações para a popularização da ciência.

A Fundaj é uma instituição de pesquisa social, que conta com a peculiaridade de poder ser analisada por suas ações nos quatro quadrantes da espiral proposta por Vogt. A pluralidade institucional é selada através da produção do conhecimento científico, por sua atividade de pesquisa; do resgate e armazenamento histórico e cultural, por seus acervos e base de dados disponibilizados em espaços virtuais e presenciais; da formação profissional, pelos programas de bolsas de pesquisa para graduação; e da pós-graduação, através dos cursos de especialização e mestrado que oferece. Conta, ainda, com a ampla divulgação do conhecimento científico e cultural através dos seus diversos equipamentos culturais, site institucional e mídia. Dessa forma, articulada com parcerias institucionais, a Fundaj produz conhecimento, ensina, disponibiliza e difunde conhecimento amplamente, contribuindo com a formação de uma sociedade crítica e capaz.

O estudo apontou que a Fundação Joaquim Nabuco tem potencial institucional de cultura científica. Além do rico acervo, possui história e reconhecimento consolidado em seus 66 anos de atividades no campo da pesquisa social. Identificaramse ações que configuram um potencial significativo de produção a ser disseminada nos diversos níveis de circulação do conhecimento entre destinadores e destinatários.

Porém, algumas dessas ações não se concretizam satisfatoriamente, entre elas a participação dos pesquisadores em eventos científicos (ver Quadro 4), as publicações, ainda dependentes das iniciativas individuais (ver Quadros 5 e 6), e a oscilação e até mesmo subutilização de recursos apontada pelo percentual de uso dos recursos para realização de eventos (Quadro 3). As revistas CES e C\&T, embora longevas, apresentam problemas em relação à periodicidade e ao aporte de infraestrutura. A classificação Qualis obtida revela suas necessidades de qualidade. Além disso, a instituição apresenta um cenário com muitas oportunidades de melhoria no quadran- 
te quatro, o qual finaliza o ciclo da cultura científica com a completa divulgação da informação científica para a sociedade.

Essa constatação é um aspecto importante deste estudo, pois a expressão “cultura científica”, adotada por Vogt (2003), pressupõe uma concepção de conhecimento científico, desde seu nascimento até sua ampla divulgação, visando ao seu uso social.

As evidências mostraram que a informação científica produzida na Dipes/Fundaj percorre o caminho delineado pela Espiral da cultura Científica (VOGT, 2003), mesmo encontrando inúmeras dificuldades e barreiras. Por tudo isso, o potencial institucional de cultura científica aponta em favor da implementação de um programa institucionalizado de cultura científica na Fundaj e para a necessidade de ações integradas entre as diversas áreas que a compõem, buscando o seu efetivo reconhecimento como locus de pesquisa, produção e disseminação do conhecimento, na perspectiva do uso social da ciência.

\section{Referências}

BENZ, Paulo André; SICSÚ, Abraham Benzaquen. Eficiência nas organizações públicas e gestão do conhecimento: otimizando sistemas. In: AGUIAR, Sylvana Maria Brandão de (Org.). Gestão pública: práticas e desafios. Recife: Bagaço, 2009. v. 3

BERLINGUET, Louis. Scientific, Technical and Industrial Culture in the World of XXIst Century. In: SCHIELE, Bernard; AMYOT, Michel; BENOIT, Claude (Coord.). When science becomes culture: world survey of scientific culture (Proceedings I). Montréal: University of Ottawa, 1994. Preface. Disponível em: <http://www.cirst.uqam.ca/pcst3/PDF/actes/When\%20science\%20becomes\%20cult ure.pdf>. Acesso em: 17 abr. 2015.

BRASIL. Fundação Joaquim Nabuco. Resolução CONDIR no 174/2014. Aprova o relatório final do Plano de desenvolvimento Institucional. Recife, 2014.

BRASIL. Lei $\mathrm{n}^{\circ} 12.527$, de 18 de novembro de 2011. Regula o acesso a informações. Diário Oficial [da] República Federativa do Brasil, Poder Executivo, Brasília, DF, 18 nov. 2011. Edição extra. Disponível em: <http://www.planalto.gov.br/ccivil_03/_ato2011-2014/2011/lei/112527.htm>. Acesso em: 17 abr. 2015. 
BRASIL. Lei $\mathrm{n}^{\circ} 770$, de 21 de julho de 1949. Abre o crédito especial de Cr\$ 2.000.000,00, para atender às despesas de comemoração do centenário de Joaquim Nabuco. Diário Oficial [da] República Federativa do Brasil, Poder Executivo, Brasília, DF, 27 jul. 1949. Seção 1, p. 10705. Disponível em:

<http://www2.camara.leg.br/legin/fed/lei/1940-1949/lei-770-21-julho-1949-363883publicacaooriginal-1-pl.html>. Acesso em: 17 abr. 2015.

BRASIL. Ministério da Ciência, Tecnologia e Informação. Estratégia Nacional de Ciência, Tecnologia e Inovação 2012-2015. Brasília: MCT\&I, 2012. Disponível em: 〈http://livroaberto.ibict.br/docs/218981.pdf〉. Acesso em: 15 abr. 2015.

BUENO, Wilson Costa. Jornalismo científico como resgate da cidadania. In: MASSARANI, Luisa; MOREIRA, Ildeu. Castro.; BRITO, Fatima. (Org.). Ciência e Público: caminhos da divulgação científica no Brasil. Rio de Janeiro: Casa da Ciência/UFRJ, 2002. p. 229-230

CALSAMIGLIA, Helena. Divulgar: itinerarios discursivos del saber. Quark: ciencia, medicina, comunicación y cultura, Barcelona, n. 7, p. 9-18, 1997.

Disponível em: <http://dialnet.unirioja.es/servlet/articulo?codigo=230242>. Acesso em: 15 abr. 2015.

CAPES. Plataforma Sucupira: Portal Qualis. 2015. Disponível em: <https://sucupira.capes.gov.br/sucupira/public/consultas/coleta/veiculoPublicacaoQu alis/listaConsultaGeralPeriodicos.jsf>. Acesso em: 20 maio 2015.

CAPES. Classificação da produção intelectual. Brasília: CAPES, 2014.

Disponível em: <http://www.capes.gov.br/avaliacao/instrumentos-deapoio/classificacao-da-producao-intelectual>. Acesso em: 15 abr. 2015.

FRESTON, Paul. Um império na província: o Instituto Joaquim Nabuco em Recife. In: MICELI, Sérgio. (Org.). História das Ciências Sociais no Brasil. 2. ed. São Paulo: Sumaré, 2001. v. 1, p. 369-417

FREYRE, Gilberto. Discurso do deputado Gilberto Freyre apresentando o projeto de criação do Instituto Joaquim Nabuco. In: BRASIL. Ministério da Educação e Saúde. Boletim do Instituto Joaquim Nabuco. Recife: IJN, 1952. v. 1, n. 1, p. 6.

FUNDAÇÃO JOAQUIM NABUCO. Relatório Anual. Recife: 2012, p. 9.

FUNDAÇÃO JOAQUIM NABUCO. Relatório de Gestão da Dipes 2003-2006. Recife: 2007a.

FUNDAÇÃO JOAQUIM NABUCO. Relatório de Gestão da Dipes 2007. Recife: $2007 b$.

FUNDAÇÃO JOAQUIM NABUCO. Relatório de Gestão da Dipes 2013. Recife: 2013. 
KREINZ, Glória; PAVAN, Crodowaldo (Org.). A espiral em busca do infinito: ensaios sobre o divulgador científico José Reis. São Paulo: USP, 1998. (Coleção Divulgação Científica, 1). p. 113

LE COADIC, Yves-François. A Ciência da Informação. 2. ed. Brasília: Briquet de Lemos Livros, 2004.

MASSARANI, Luisa. A divulgação científica no Rio de Janeiro: algumas reflexões sobre a década de 20. 1998. Dissertação (Mestrado em Ciência da Informação) - Instituto Brasileiro de Informação em C\&T e Escola de Comunicação da Universidade Federal do Rio de Janeiro, Rio de Janeiro, 1998. Disponível em: $\langle$ http://www.casadaciencia.ufrj.br/Publicacoes/Dissertacoes/Massarani_tese.pdf $>$. Acesso em: 17 abr. 2015.

MUELLER, Suzana P. M. Popularização do Conhecimento Científico. DataGramaZero, Rio de Janeiro, v. 3, n. 2, abr., 2002. Disponível em: <http://www.dgz.org.br/abr02/Art_03.htm>. Acesso em: 17 abr. 2015.

NASCIMENTO, Tatiana Galieta. Definições de Divulgação Científica por jornalistas, cientistas e educadores em ciências. Revista Ciência em Tela, Rio de Janeiro, v. 1, n. 2, 2008. Disponível em:

$\langle$ http://www.cienciaemtela.nutes.ufrj.br/artigos/0208nascimento.pdf $>$. Acesso em: 17 abr. 2015.

REIS, José. Educação é investimento. São Paulo: IBRASA, 1968.

UNIVERSIDADE ESTADUAL DE CAMPINAS. Laboratório de Estudos Avançados em Jornalismo. Histórico. 2015. Disponível em:

<http://www.labjor.unicamp.br/historico.htm>. Acesso em: 17 abr. 2015.

VOGT, Carlos. SAPO (Science Authomatic Press Observer): construindo um barômetro da ciência e tecnologia na mídia. In: VOGT, Carlos (Org.). Cultura Científica: desafios. São Paulo: Fapesp, 2006. p. 84-130

VOGT, Carlos. A espiral da cultura científica. ComCiência, Campinas, n. 45, jul. 2003. Disponível em:

<http://www.comciencia.br/reportagens/cultura/cultura01.shtml >. Acesso em: 15 abr. 2015. 


\title{
Science communication: the social use of the product of scientific studies in the Joaquim Nabuco Foundation
}

\begin{abstract}
This research identified the path covered by the scientific information produced in the Social Research Directorate (Dipes) of the Joaquim Nabuco Foundation (Fundaj), from 2004 to 2013, noting the channels and formats that were used, as well as the actors involved. The study took advantage of the spiral created by Vogt (2003) to represent the environment of scientific culture in order to analyze Fundaj's context through the examination of institutional documents and semistructured interviews with researchers and managers of the institution. Although Fundaj counts with institutional potential for scientific culture, this study found that part of the activities is carried out by individual initiatives. These findings are important as the "scientific culture" expression adopted by Vogt (2003) requires the construction of scientific knowledge, from its origin to its wide dissemination, aiming at its social use.
\end{abstract}

Keywords: Scientific communication. Scientific culture. Fundação Joaquim Nabuco.

Recebido em: 22/04/2015

Aceito em: 06/07/2015

${ }^{1}$ BUENO, Wilson C. Jornalismo científico: conceitos e funções. Ciência e Cultura, Campinas, v. 37, n. 9, p. 1420-1427, 1985.

2 REIS, José. Educação é investimento. São Paulo: IBRASA, 1968.

${ }^{3}$ No original: "El proceso por el cual se hace llegar a un público no especializado y amplio el saber producido por especialistas en una disciplina científica.”. 